\title{
Organocatalytic Enantioselective Synthesis of Pyrazoles Bearing a Quaternary Stereocenter
}

\author{
Carlos Vila, ${ }^{*[a]}$ Fares Ibrahim Amr, ${ }^{[a]}$ Gonzalo Blay, ${ }^{[a]}$ M. Carmen Muñoz, ${ }^{[b]}$ and José R. Pedro*[a]
}

\begin{abstract}
An efficient one-pot asymmetric synthesis of pyrazoles bearing a chiral quaternary stereocenter has been developed. Quinine derived thiourea catalyzed the enantioselective addition of pyrazolones to isatin-derived ketimines providing the corresponding acetylated pyrazoles after in situ treatment with $\mathrm{Ac}_{2} \mathrm{O} / \mathrm{Et}_{3} \mathrm{~N}$. The corresponding pyrazoles were afforded with high yields and excellent enantioselectivities.
\end{abstract}

\section{Introduction}

Five-membered nitrogen heterocycles have been recognized as privileged structural frameworks for the development of molecules with biological and pharmaceutical properties. Among the various five-membered nitrogen heterocycles, pyrazole, containing two adjacent nitrogen atoms, represent an important class of aromatic heterocycle that recently have attracted huge attention because of the wide applications in medicinal, pharmaceutical, agrochemical or coordination chemistry. ${ }^{[1]}$ For example, pyrazole ring is a scaffold present in blockbuster drugs such celecoxib and sildenafil, or in a proposed drug such remogliflozin etabonate ${ }^{[2]}$ (Figure 1).
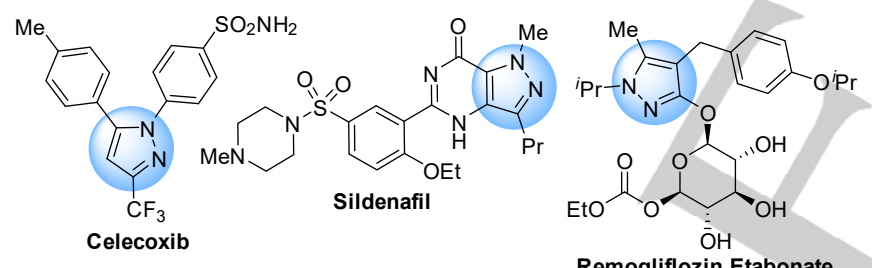

Figure 1. Representative pharmaceutical active pyrazoles.

In view of the great importance of the pyrazole skeleton, the asymmetric synthesis of pyrazoles bearing stereocenters have become an attractive goal and many and efficient synthetic approaches have been established. ${ }^{[3]}$ Among them, the addition of pyrazolone to different electrophiles and posterior acetylation or annulation represents a valuable methodology. ${ }^{[4,5]}$ Nevertheless, 1,2-addition reactions of pyrazolone for the

[a] Dr. C. Vila, Fares Ibrahim Amr, Prof. Dr. G. Blay, Prof. Dr. J. R. Pedro

Departament de Química Orgànica, Facultat de Química, Universitat de València, Dr. Moliner 59, 46100 Burjassot, València (Spain) Fax: (+34) 963544328

E-mail: carlos.vila@uv.es, jose.r.pedro@uv.es

[b] Prof. Dr. M. C. Muñoz

Departament de Física Aplicada, Universitat Politècnica de València, Camino de Vera s/n, 46022 València (Spain)

Supporting information for this article is given via a link at the end of the document.((Please delete this text if not appropriate)) synthesis of chiral pyrazoles have not been described as far as we know.

On the other hand, the oxindole moiety constitutes an important and privileged scaffold present in numerous natural products and pharmacologically active compounds. ${ }^{[6]}$ One way to prepare enantioenriched amino oxindole adducts is the asymmetric addition of nucleophiles to isatin-derived ketimines. ${ }^{[7]}$ Given the relevance of pyrazole and amino oxindoles ${ }^{[8]}$ it was anticipated that the incorporation of both structural motifs into one molecule could result in a novel chiral pyrazoles bearing a quaternary stereocenter with potentially interesting biological properties (Scheme 1). ${ }^{[9]}$

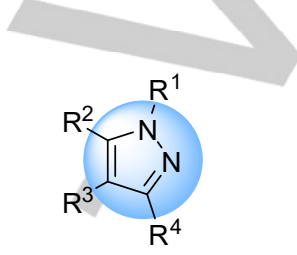

Pyrazole: important pharmacophore

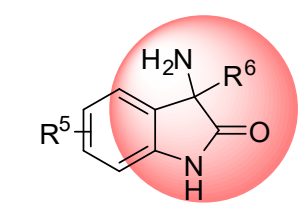

Amino oxindole: biologically important molecule

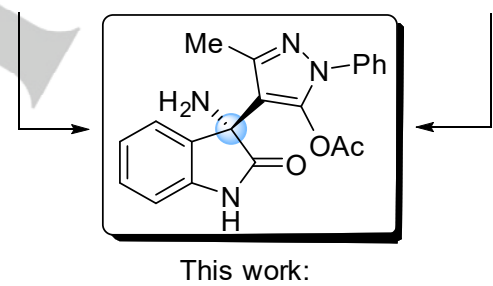

synthesis of novel chiral

pyrazole-amino oxindole adducts

(potential biological activity)

Scheme 1. Relevance of pyrazole-amino oxindole adducts.

As a part of our ongoing interest in the asymmetric synthesis of nitrogen aromatic heterocycles bearing a quaternary stereocenter ${ }^{[10]}$ and the synthesis of optically pure amino oxindole compounds, ${ }^{[1]}$ here we described the highly enantioselective addition of pyrazolone to isatin-derived ketimines, ${ }^{[12]}$ providing a novel pyrazole adducts after acetylation of the pyrazolone moiety.

\section{Results and Discussion}

We initiated our studies by evaluating the reaction between isatin-derived $\mathrm{N}$-Boc ketimine $1 \mathrm{a}$ and 5-methyl-2-phenyl-2,4dihydro-3H-pyrazol-3-one (2a) in the presence of a series of bifunctional organocatalysts. ${ }^{[13]}$ We thought in the use of bifunctional organocatalysts with a hydrogen bonding donor moiety in order to activate the electrophile (the isatin-derived $\mathrm{N}$ Boc ketimine), and a tertiary amine responsible for the activation of the nucleophile (the pyrazolone). As shown in Table 1, when 5 mol\% of quinine (Cat-1) was used in toluene, full conversion to 
the addition product was obtained within 5 hours. However, the analysis by NMR spectroscopy indicated that the addition product was a mixture of tautomers and diastereomers. Fortunately, this mixture could be in situ acetylated with $\mathrm{Et}_{3} \mathrm{~N} / \mathrm{Ac}_{2} \mathrm{O}$, affording the corresponding pyrazole-amino oxindole adduct $3 \mathrm{aa}$ in $82 \%$ yield, but with low enantioselectivity ( $29 \%$ ee, entry 1 Table 1$)$.

Table 1. Optimization of the reaction conditions. obtained with poor enantioselectivities (entry 3 and 4, respectively). To our delight, quinine derived thiourea Cat-5, ${ }^{[14]}$ exhibited excellent reactivity with high enantioselectivity, affording product 3aa with an excellent enantiomeric excess of $99 \%$ (entry 5). Quinine derived squaramide Cat-6, ${ }^{[15]}$ could promote the reaction with high stereocontrol and good yield, although with slightly lower enantiomeric excess than Cat-5 (entry 6). Interestingly, when Takemoto's catalyst ${ }^{[16]}$ Cat-7 was used product 3aa was obtained with high yield and excellent enantioselectivity ( $98 \%$ ee), but to the opposite enantiomer (entry 7 ). With thiourea Cat-4, we screened the catalyst loading and we found that we could decrease the catalyst loading to only $1 \mathrm{~mol} \%$ without compromising the yield and enantiomeric excess (entry 9).

With the optimized reaction conditions stablished, the scope of the reaction was explored with respect to both the isatin-derived $N$-Boc ketimines and the pyrazolone. First we tested different isatin-derived ketimines with pyrazolone 2a (Scheme 2). Initially, $\mathrm{N}$-substitution of the oxindole nitrogen was evaluated (Scheme 2, 3aa-3da). Groups such as benzyl, methyl or allyl were well tolerated providing the corresponding adducts with excellent enantioselectivities (98-99\% ee). In addition, a non-protected free $\mathrm{NH}$ on the oxindole ring was also tolerated (3da), which allows for facile potential $\mathrm{N}$-substitutions on demand. Electron-donating $(\mathrm{MeO})$ or electron-withdrawing $\left(\mathrm{Cl}\right.$ or $\left.\mathrm{NO}_{2}\right)$, were tolerated at the 5 position of the isatin-derived ketimine, affording the corresponding products (3ea-3ga) with good yields and high stereocontrol. Moreover, ketimines with substituents at 6 or 7 positions reacted smoothly, providing pyrazoles 3ha and 3ia with very good results.

\begin{tabular}{lllll}
\hline Entry $^{[a]}$ & Catalyst $(\mathrm{x} \mathrm{mol} \%)$ & $\mathrm{t}(\mathrm{h})$ & ${\text { Yield }(\%)^{[\mathrm{b}]}}$ & $\mathrm{Ee}(\%)^{[\mathrm{cc}]}$ \\
\hline 1 & Cat-1 $(5 \mathrm{~mol} \%)$ & 5 & 82 & 29 \\
2 & Cat-2 $(5 \mathrm{~mol} \%)$ & 5 & 92 & $15^{[\mathrm{d}]}$ \\
3 & Cat-3 (5 mol\%) & 7 & 74 & $31^{[\mathrm{d]}]}$ \\
4 & Cat-4 (5 mol\%) & 5 & 94 & $8^{[\mathrm{d}]}$ \\
5 & Cat-5 (5 mol\%) & 2 & 82 & 99 \\
6 & Cat-6 (5 mol\%) & 2 & 92 & 97 \\
7 & Cat-7 (5 mol\%) & 3,5 & 91 & $98^{[\mathrm{dd}]}$ \\
8 & Cat-5 (2 mol\%) & 3,5 & 98 & 99 \\
9 & Cat-5 (1 mol\%) & 3,5 & 95 & 99 \\
\hline
\end{tabular}

[a] Reaction conditions: $1 \mathrm{a}(0.1 \mathrm{mmol}), \mathbf{2 a}(0.1 \mathrm{mmol})$ and catalyst $(\mathrm{x} \mathrm{mol} \%)$ in $1 \mathrm{~mL}$ of toluene at $\mathrm{rt}$. [b] Isolated yield after column chromatography. [c] Determined by HPLC using chiral stationary phase. [d] Opposite enantiomer was obtained.

With quinidine (Cat-2, entry 2), a slightly better yield was obtained (92 \%), but a lower enantiomeric excess of the opposite enantiomer was observed ( $15 \%$ ee). Cupreine derivatives Cat-3 and Cat-4, could catalyze the reaction but product 3aa was

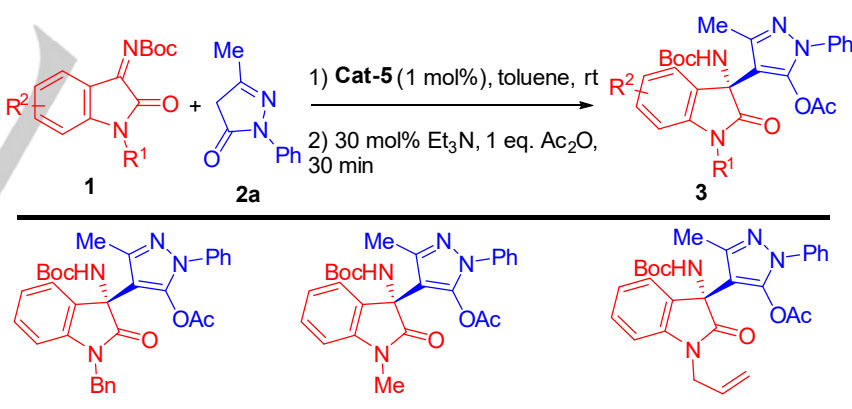

3aa, $95 \%$ yield, $99 \%$ ee $\quad 3$ ba, $83 \%$ yield, $98 \%$ ee

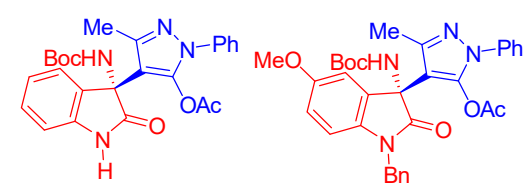

3da, $97 \%$ yield, $96 \%$ ee<smiles>CC(=O)Oc1c([C@]2(N)C(=O)N(Cc3ccccc3)c3ccc([N+](=O)[O-])c(Br)c32)c(C)nn1-c1ccccc1</smiles>

$\mathrm{Bn}$

3ga, $65 \%$ yield, $97 \%$ ee 3ea, $84 \%$ yield, $98 \%$ ee

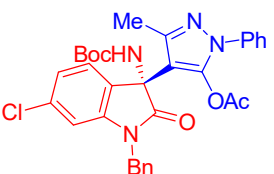

3ha, $78 \%$ yield, $98 \%$ ee 3ca, $88 \%$ yield, $99 \%$ ee

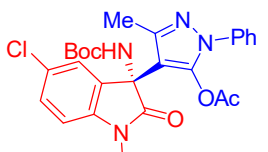

$\mathrm{Bn}$

3fa, $70 \%$ yield, $98 \%$ ee

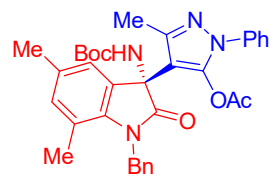

3ia, $98 \%$ yield, $95 \%$ ee
Scheme 2. Scope for different isatin derived ketimines: 1 (0.05 mmol), 2a (0.051 $\mathrm{mmol}$ ) and Cat-5 (1 mol\%) in $1 \mathrm{~mL}$ of toluene. Isolated yields after column chromatography. Enantiomeric excesses were determined by HPLC using chiral stationary phase. 
Different pyrazolones 2 were also evaluated in the reaction with isatin-derived $N$-Boc ketimine 1a (Scheme 3). The reaction proceeded efficiently with pyrazolones with diverse substituents ( $\mathrm{MeO}$ or $\mathrm{Cl}$ ) on the $\mathrm{N}$-aryl group. Moreover, when 2,5-diphenylpyrazolone was tested the corresponding product $3 \mathrm{ad}$ was afforded with $98 \%$ yield and $97 \%$ ee. Once we studied different substituted pyrazolones, we turned our attention on the trapping agent for the enol form of the pyrazolone moiety. After the addition of pyrazolone 2a to the ketimine 1a was completed, benzoyl chloride $(\mathrm{BzCl})$ and $\mathrm{Et}_{3} \mathrm{~N}$ were added to the reaction mixture and the corresponding chiral pyrazole 3 af was afforded with excellent yield $(97 \%)$ and enantioselectivity (99\% ee). Similarly, when mesyl chloride $(\mathrm{MsCl})$ was used for the second step, the corresponding mesylated pyrazole 3ag was obtained with high yield and enantiomeric excess. On the contrary, when ethyl chloroformate was used, the reactivity was lower and the product 3ah was gained with lower yield (62\%), although with excellent enantioselectivity ( $98 \%$ ee).

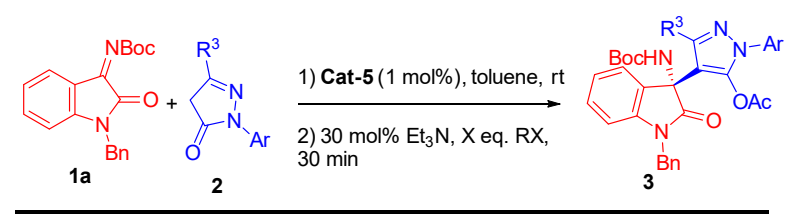

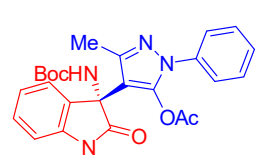

$\mathrm{Bn}$

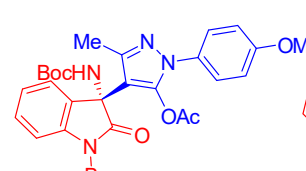

$\mathrm{Bn}$

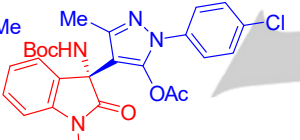

$\mathrm{Bn}$ 3aa, ${ }^{\text {a }} 95 \%$ yield, $99 \%$ ee $\quad$ 3ab, $^{\text {a }} 85 \%$ yield, $98 \%$ ee $\quad$ 3ac, ${ }^{\text {a }} 96 \%$ yield, $96 \%$ ee<smiles>CC(=O)Oc1c([C@]2(Nc3ccccc3)C(=O)N(Cc3ccccc3)c3ccccc32)nn(-c2ccccc2)c1-c1ccccc1</smiles><smiles>CC(=O)Oc1c([C@@]2(Nc3ccccc3)C(=O)N([O+])c3ccccc32)c(-c2ccccc2)nn1-c1ccccc1</smiles>

$\mathrm{Bn}$

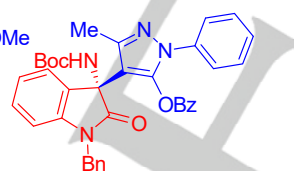$$
\mathrm{Bn}
$$

3ad, ${ }^{\text {a }} 98 \%$ yield, $97 \%$ ee $\quad 3$ ae, ${ }^{\text {a }} 95 \%$ yield, $98 \%$ ee $\quad$ 3af, ${ }^{\text {b }} 97 \%$ yield, $99 \%$ ee<smiles>COc1c([C@]2(N)C(=O)Nc3ccccc32)c(C)nn1-c1ccccc1</smiles>

$\mathrm{Bn}$

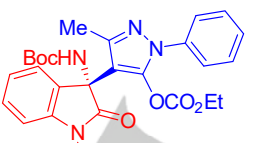

$\mathrm{Bn}$

3ag, ${ }^{\mathrm{c}} 92 \%$ yield, $94 \%$ ee $\quad 3$ ah, ${ }^{\mathrm{d}} 62 \%$ yield, $98 \%$ ee

Scheme 3. Scope for different pyrazolones: 1a $(0.05 \mathrm{mmol}), 2(0.051 \mathrm{mmol})$ and Cat-5 (1 mol\%) in $1 \mathrm{~mL}$ of toluene. Isolated yields after column chromatography. Enantiomeric excesses were determined by HPLC using chiral stationary phase. ${ }^{a} \mathrm{Ac}_{2} \mathrm{O}$ (1 eq.) was used for the second step. ${ }^{\mathrm{b}} \mathrm{BzCl}(2$ eq.) was used for the second step. ${ }^{\mathrm{C}} \mathrm{MsCl}$ (2 eq.) was used for the second step. ${ }^{\mathrm{d}} \mathrm{CICO}_{2} \mathrm{Et}$ (2 eq.) was used for the second step.

The absolute configuration of the stereogenic center in compound 3ha was determined to be $(R)$ on the basis of X-ray crystallographic analysis (Figure 2); ${ }^{[17]}$ the configuration of the other products 3 was assigned on the assumption of a uniform mechanistic pathway.

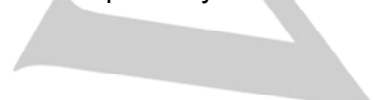

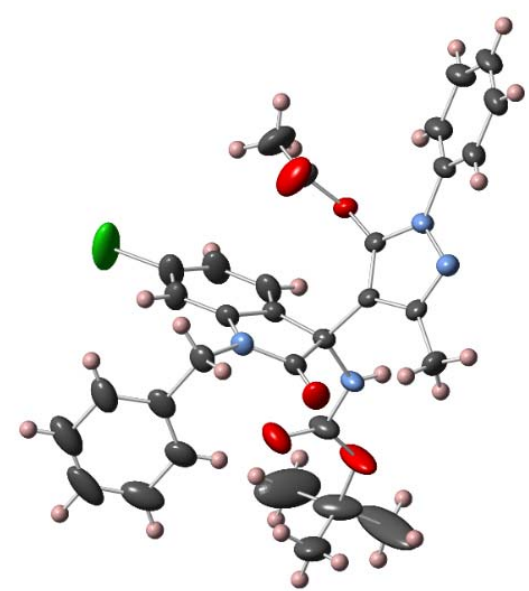

Figure 2. X-ray crystal structure of 3 ha.

A plausible transition-state model for the enantioselective reaction is shown in Scheme 4. The thiourea catalyst is responsible for the preorientation and activation of the substrates acting as a bifunctional organocatalyst. The isatin-derived $\mathrm{N}$-Boc ketimine is activated upon formation of hydrogen bonds between the thiourea moiety and the $\mathrm{N}-\mathrm{Boc}$ and the carbonyl groups of the oxindole, while at the same time the pyrazolone undergoes nucleophilic activation by hydrogen bonding with the quinuclidine moiety of the catalyst. ${ }^{[5--b, 18]}$ The pyrazolone 2 will attack to the Re-face of the ketimine, thus accounting for the observed enantioselectivity. The corresponding acetylated pyrazoles $\mathbf{3}$ are obtained after in situ treatment with $\mathrm{Ac}_{2} \mathrm{O} / \mathrm{Et}_{3} \mathrm{~N}$.

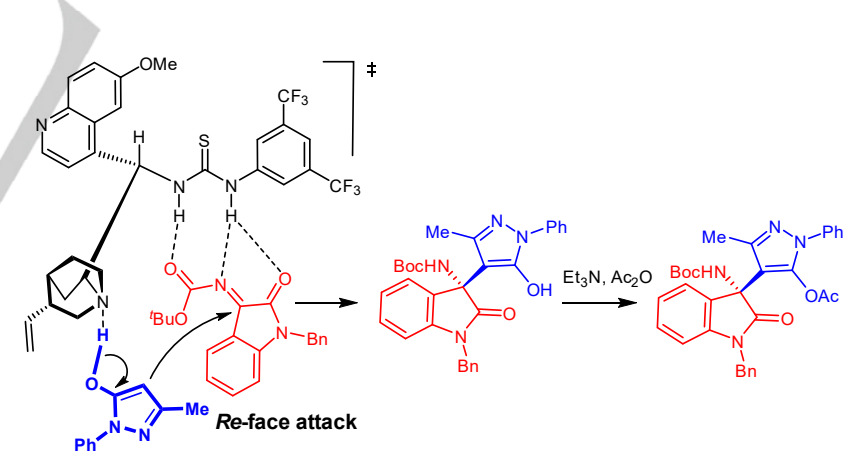

Scheme 4. Plausible transition-state model.

\section{Conclusions}

In conclusion, we have successfully developed a thiourea derived quinine catalyzed enantioselective addition of pyrazolones to isatin-derived ketimines. The corresponding acetylated pyrazoles bearing a tetrasubstituted stereocenter were obtained in good yields (up to $98 \%$ ) and excellent enantioselectivities (up to $99 \%$ ee). Furthermore, the enol form of the pyrazolone product could be trapped with different agents as $\mathrm{Ac}_{2} \mathrm{O}, \mathrm{BzCl}, \mathrm{MsCl}$ or $\mathrm{ClCO}_{2} \mathrm{Et}$. This organocatalytic methodology provides access to chiral 
heterocyclic compounds bearing pyrazole and aminooxindole moieties, which possess potential pharmaceutical activities.

\section{Experimental Section}

\section{General procedure for the enantioselective reaction}

A test tube containing ketimine $1 \mathbf{a}(0.1 \mathrm{mmol})$, pyrazolone $\mathbf{2 a}(0.1 \mathrm{mmol})$ and thiourea Cat-5 $(0.6 \mathrm{mg}, 0.001 \mathrm{mmol})$ was purged with $\mathrm{N}_{2}$. Then, 1.0 $\mathrm{mL}$ of toluene was added and the mixture stirred at room temperature until the reaction was complete (TLC). After the reaction was complete, $\mathrm{Ac}_{2} \mathrm{O}$ $(0.1 \mathrm{mmol})$ and $\mathrm{Et}_{3} \mathrm{~N}(0.03 \mathrm{mmol})$, were added and the reaction mixture was stirred for 30 additional minutes. Finally, the reaction mixture was directly poured to the column chromatography, using hexane:EtOAc (95:5) as eluent to afford product 3aa.

\section{Acknowledgements}

Financial support from the MINECO (Gobierno de España; CTQ2013-47494-P) and from Generalitat Valenciana (ISIC2012/001) is gratefully acknowledged. C.V. thanks MINECO for a JdC contract. Access to NMR, MS and X-ray facilities from the Servei central de suport a la investigació experimental (SCSIE)-UV is also acknowledged.

Keywords: asymmetric catalysis • organocatalysis • pyrazoles • isatin-derived ketimines $\bullet$ quaternary stereocenter

[1] a) A. Schmidt, A. Dreger, Curr. Org. Chem, 2011, 15, 1423-1463; b) S Fustero, M. Sánchez-Roselló, P. Barrio, A. Simón-Fuentes, Chem. Rev., 2011, 111, 6984-7034; c) V. Kumar, K. Kaur, G. K.; Gupta, A. K. Sharma, Eur. J. Med. Chem., 2013, 69, 735-753.

[2] Y. Fujimori, K. Katsuno, I. Nakashima, Y. Ishikawa-Takemura, H. Fujikura, M. Isaji, J. Pharmacol. Exp. Ther., 2008, 327, 268-276.

[3] a) X. Hu, B. Gao, Y. Chu, W. Li, X. Liu, L. Lin, X. Feng, Chem. Eur. J. 2012, 18, 3473-3477; b) M. C. Pérez-Aguilar, C. Valdés, Angew. Chem. 2015, 127, 13933-13937; Angew. Chem. Int. Ed. 2015, 54, 13729-13733 c) S.-J. Lee, J.-Y. Bae, C.-W. Cho, Eur. J. Org. Chem. 2015, 6495-6502 d) Q. Lin, D. Meloni, Y. Pan, M. Xia, J. Rodgers, S. Shepard, M. Li, L. Galya, B. Metcalf, T.-Y. Yue, P. Liu, J. Zhou, Org. Lett. 2009, 11, 19992002; e) C. M. Park, D. J. Jeon, Org. Biomol. Chem. 2012, 10. 26132620; f) G. Blay, I. Fernández, E. Molina, M. C. Muñoz, J. R. Pedro, C. Vila, Tetrahedron, 2006, 62, 8069-8076. g) A. M. Haydl, K. Xu, B. Breit, Angew. Chem. 2015, 127, 7255-7259; Angew. Chem. Int. Ed. 2015, 54, 7149-7153; h) J. Zhang, Y. Zhang, X. Liu, J. Guo, W. Cao, L. Lin, X. Feng Adv. Synth. Catal. 2014, 356, 3545-3550; i) Z.-C. Geng, J.-X. Zhang, N. Li, J. Chen, X.-F. Huang, S.-Y. Zhang, H.-Y. Li, J.-C. Tao, X.-W. Wang, Tetrahedron 2014, 70, 417-426.

[4] For a review, see: a) P. Chauan, S. Mahajan, D. Enders, Chem. Commun. 2015, 51, 12890-12907. For selected examples of asymmetric pyrazolone additions: b) Y.-H. Liao, W.-B. Chen, Z.-J. Wu, X.-L. Du, L.F. Cun, X.-M. Zhang, W.-C. Yuan, Adv. Synth. Catal. 2010, 352, 827832; c) Z. Wang, Z. Yang, D. Chen, X. Liu, L. Lin, X. Feng, Angew. Chem. 2011, 123, 5030-5034; Angew. Chem. Int. Ed. 2011, 50, 4928-4932; d) Z. Yang, Z. Wang, S. Bai, X. Liu, L. Lin, X. Feng, Org. Lett. 2011, 13, 596-599; e) Z. Wang, Z. Chen, S. Bai, W. Lei, X. Liu, L. Lin, X. Feng, Angew. Chem., 2012, 124, 2830-2833; Angew. Chem. Int. Ed. 2012, 51 2776-2779; f) Z.-L. Tao, W.-Q. Zhang, D.-F. Chen, A. Adele, L.-Z. Gong, J. Am. Chem. Soc. 2013, 135, 9255-9258; g) D. Hack, A. B. Dîrr, K. Deckers, P. Chauhan, N. Seling, L. Rîbenach, L. Mertens, G. Raabe, F.
Schoenebeck, D. Enders, Angew. Chem. 2016, 128, 1829-1832; Angew. Chem. Int. Ed. 2016, 55, 1797-1800. h) X. Han,W. Yao,T. Wang,Y. R. Tan, Z. Yan, J. Kwiatkowski, Y. Lu, Angew. Chem. 2014, 126, 57495753; Angew. Chem. Int. Ed. 2014, 53, 5643-5647.

[5] a) J. H. Li, D.-M. Du, Org. Biomol. Chem. 2015, 13, 5636-5645; b) D. Hack, P. Chauan, K. Deckers, Y. Mizutani, G. Raabe, D. Enders, Chem Commun. 2015, 51, 2266-2269; c) D. Enders, A. Grossmann, B. Gieraths, M. Düzdemir, C. Merkers, Org. Lett., 2012, 14, 4254-4257; d) S. Gogoi, C.-G. Zhao, Tetrahedron Lett., 2009, 50, 2252-2255; e) S. R. Yetra, S. Mondal, E. Suresh, A. T. Biju, Org. Lett. 2015, 17, 2030-2037; f) Y. Zhang, S. Wu, S. Wang, K. Fang, G. Dong, N. Liu, Z. Miao, J. Yao, J. Li, W. Zhang, C. Sheng, W. Wang, Eur. J. Org. Chem. 2015, 2030-2037. g) S. Wang, C. Rodriguez-Escrich, M. A. Pericàs, Org. Lett., 2016, 18, 556559 ; h) N. Kumarswamyreddy, V. Kesavan, Org. Lett., 2016, 18,13541357

[6] For a reviews, see: a) R. Dalpozzo, G. Bartoli, G. Bencivenni, Chem. Soc Rev., 2012, 41, 7247-7290; b) F. Zhou, Y.-L. Liu, J. Zhou, Adv. Synth. Catal., 2010, 352, 1381-1407; c) C. V. Galliford, K. A. Scheidt, Angew. Chem., 2007, 119, 8902-8912; Angew. Chem., Int. Ed., 2007, 46, 87488758; d) C. Marti, E. M. Carreira, Eur. J. Org. Chem., 2003, 2209-2219; e) H. Lin, S. J. Danishefsky, Angew. Chem., 2003, 115, 38-53; Angew. Chem., Int. Ed., 2003, 42, 36-51; f) B. M. Trost, M. K. Brennan, Synthesis, 2009, 3003-3025; for recent examples of natural oxindoles, see: g) X. Xu, X. Zhang, X. Nong, X. Wei, W. Qi, Tetrahedron 2015, 72, 610-615; h) M.X., Sun, H.-H., Gao, J. Zhao, L. Zhang, K. Xiao Tetrahedron Lett. 2015, 56, 6194-6197; for pharmacologically active oxindoles, see: i) J. Walker, R. W. Daisley, A. H. Beckett, J. Med. Chem. 1970, 13, 983-985; for the enantioselective synthesis of oxindoles containing quaternary stereocenters j) L. Zong, S. Du, K. F. Chin, C. Wang, C.-H. Tan, Angew Chem. Int. Ed. 2015, 54, 9390-9393.

[7] a) P. Chauhan, S. S. Chimni, Tetrahedron: Asymmetry, 2013, 24, 343356; b) J. Kaur, S. S. Chimni, S. Mahajan, A. Kumar, RSC Adv. 2015, 5, 52481-52496; c) J.-S. Yu, F. Zhou, Y.-L. Liu, J. Zhou, Synlett, 2015, 26 2491-2504. Selected examples: d) J. Zhao, B. Fang, W. Luo, X. Hao, X. Liu, L. Lin, X. Feng, Angew. Chem., 2015, 127, 243-246, Angew. Chem. Int. Ed. 2015, 54, 241-244; e) O. D. Engl, S. P. Fritz, H. Wennemers, Angew. Chem., 2015, 127, 8311-8315; Angew. Chem., Int. Ed. 2015, 54, 8193-8197; f) H. M. Zhang, Z. H. Gao, S. Ye, Org. Lett. 2014, 16, 3079 3081; g) N. Hara, S. Nakamura, M. Sano, R. Tamura, Y. Funahashi, N. Shibata, Chem. Eur. J. 2012, 18, 9276-9280; h) W. Yan, D. Wang, J. Feng, P. Li, D. Zhao, R. Wang, Org. Lett. 2012, 14, 25122515; i) D. Wang, J. Liang, J. Feng, K. Wang, Q. Sun, L. Zhao, D. Li, W. Yan, R. Wang, Adv. Synth. Cat. 2013, 355, 548-558; j) Y.-L. Liu, J. Zhou, Chem. Commun. 2013, 49, 4421-4423; k) T. Arai, E. Matsumura, H. Masu, Org. Lett. 2014, 16, 2768-2771; I) S. Nakamura, K. Hyodo, M. Nakamura, D. Nakane, H. Masuda, Chem. Eur. J. 2013, 19, 7304-7309; m) J. Xu, C. Mou, T. Zhu, B.-A. Song, Y. R. Chi, Org. Lett. 2014, 16, 3272-3275; n) J. Feng, W. Yan, D. Wang, P. Li, Q. Sun, R. Wang, Chem. Commun. 2012, 48, 8003-8005; o) K. Zhao, T. Shu, J. Jia, G. Raabe, D. Enders, Chem. Eur. J. 2015, 21, 3933-3936.

[8] a) G Decaux, A. Soupart, G. Vassart, Lancet 2008, 371, 1624-1632; b) T. Shimazaki, M. lijima, S. Chaki, Eur. J. Pharmacol. 2006, 543, 63-67; c) K. Bernard, S. Bogliolo, J. Ehrenfeld, Br. J. Pharmacol. 2005, 144, 1037-1050; c) M. Rottmann, C. McNamara, B. S. K. Yeung, M. C. S. Lee, B. Zou, B. Russell, P. Seitz, D. M. Plouffe, N. V. Dharia, J. Tan, S. B. Cohen, K. R. Spencer, G. Gonzalez-Paez, L. Lakshiminarayana, A. Goh, R. Suwanarusk, T. Jegla, E. K. Schmitt, H.-P. Beck, R. Brun, F. Nosten, L. Renia, V. Dartois, T. H. Keller, D. A. Fidock, E. A. Winzeler, T. T. Diagana, Science, 2010, 329, 1175-1180.

[9] For reviews of hybrid-based drugs, see: a) S. Vandekerckhove, M. D'hooghe, Bioorg. Med. Chem. 2015, 23, 5098-5119, b) S. Sandhu, Y. Bansal, O. Silakari, G. Bansal, Bioorg. Med. Chem. 2014, 22, 3806-3814 c) C. Viegas-Junior, A. Danuello, V. da Silva Bolzani, E. J. Barreiro, C. A. Fraga, Curr. Med. Chem., 2007, 14, 1829-1852; d) S. B. Tsogoeva, Mini Rev. Med. Chem. 2010, 10, 773-793; e) M. Decker, Curr. Med. Chem. 
2011, 18, 1464-1475; f) J. J. Walsh, A. Bell, Curr. Pharm. Des. 2009, 15 2970-2985.

[10] a) G. Blay, I. Fernández, M. C. Muñoz, J. R. Pedro, A. Recuenco, C. Vila J. Org. Chem., 2011, 76, 6286-6294; b) G. Blay, I. Fernández, A Monleón, J. R. Pedro, C. Vila, Org. Lett., 2009, 11, 441-444; c) M. Holmquist, G. Blay, M. C. Muñoz, J. R. Pedro, Org. Lett., 2014, 16, $1204-$ 1207.

[11] a) M. Holmquist, G. Blay, J. R. Pedro, Chem. Commun. 2014, 50, 9309 9312; b) M. Montesinos-Magraner, C. Vila, R. Cantón, G. Blay, I. Fernández, M. C. Muñoz, J. R. Pedro, Angew. Chem., 2015, 127, 64186422; Angew. Chem. Int. Ed. 2015, 54, 6320-6324; I) M. Holmquist, G. Blay, M. C. Muñoz, J. R. Pedro, Adv. Synth. Catal. 2015, 357, 3857-3862.

[12] For an elegant organocatalytic addition of pyrazolones to isatin-derived ketimines and a subsequent diastereoselective fluorination of the pyrazolone moiety, see: X. Bao, B. Wang, L. Cui, G. Zhu, Y. He, J. Qu and Y. Song, Org. Lett. 2015, 17, 5168-5171.

[13] a) S.-K. Tian, Y.-G. Chen, J. F. Hang, L. Tang, P. McDaid, L. Deng, Acc. Chem. Res. 2004, 37, 621-631; b) T. Marcelli, H. Hiemstra, Synthesis 2010, 1229-1279; c) S. Connon, Chem. Eur. J. 2006, 12, 5418-5427; d) P. Chauchan, S. Mahajan, U. Kaya, D. Hack, D. Enders, Adv. Synth. Catal. 2015, 357, 253-281; e) Z. G. Zhang, P. R. Schreiner, Chem. Soc.
Rev. 2009, 38, 1187-1198; f) J. Aleman, A. Parra, H. Jiang, K. A Jørgensen, Chem. Eur. J. 2011, 17, 6890-6899; g) A. G. Doyle, E. N Jacobsen, Chem. Rev. 2007, 107, 5713; h) S. J. Connon, Chem. Commun. 2008, 2499; i) Y. Takemoto, Chem. Pharm. Bull. 2010, 58, 593; j) T. J. Auvil, A. G. Schafer, A. E. Mattson, Eur. J. Org. Chem. 2014, 2633.

[14] For seminal works using Cinchona alkaloids based thiourea catalysts: a) B. Vakulya, S. Varga, A. Csampai, T. Soós, Org. Lett. 2005, 7, 19671969; b) g) J. Ye, D. J. Dixon, P. S. Hynes, Chem. Commun. 2005, 44814483; c) S. H. McCooey, S. J. Connon, Angew. Chem., 2005, 117, 65256528; Angew. Chem. Int. Ed. 2005, 44, 6367-6370.

[15] For seminal work using Cinchona alkaloids based squaramide: J. P. Malerich, K. Hagihara, V. H. Rawal, J. Am. Chem. Soc. 2008, 130 14416-14417.

[16] T. Okino, Y. Hoashi, Y. Takemoto, J. Am. Chem. Soc. 2003, 125, 12672 12673.

[17] CCDC-1463856 (for 3ha) contains the supplementary crystallographic data. These data can be obtained free of charge from The Cambridge Crystallographic Data Centre via www.ccdc.cam.ac.uk/data request/cif.

[18] P. Kumari, S. Barik, N. H. Khan, B. Ganguly, R. I. Kureshy, S. H. R Abdiab, H. C. Bajaj, RSC Adv., 2015, 5, 69493-69501. 
Entry for the Table of Contents (Please choose one layout)

Layout 2:

Asymmetric Catalysis*

Carlos Vila, ${ }^{*[a]}$ Fares Ibrahim Amr, ${ }^{[a]}$ Gonzalo Blay, ${ }^{[a]}$ M. Carmen Muñoz, ${ }^{[b]}$ and José R. Pedro*

Page No. - Page No.

Organocatalytic Enantioselective Synthesis of Pyrazoles Bearing a Quaternary Stereocenter 